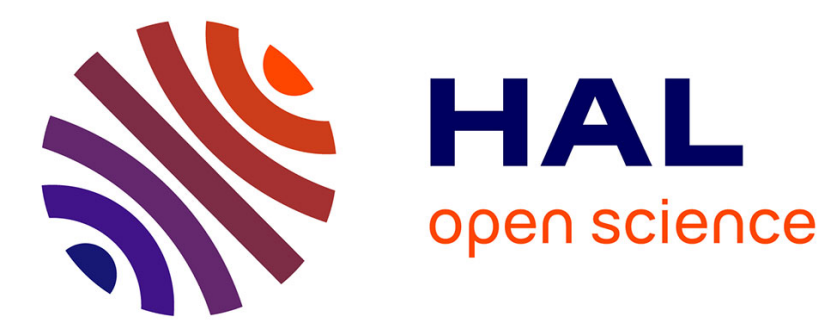

\title{
CARACTÉRISATION DU SANG PAR ULTRASONS HAUTES FRÉQUENCES
}

Malika Toubal, Edouard Radziszewski, M. Asmani, Mohamed Ourak, Bertrand Nongaillard

\section{> To cite this version:}

Malika Toubal, Edouard Radziszewski, M. Asmani, Mohamed Ourak, Bertrand Nongaillard. CARACTÉRISATION DU SANG PAR ULTRASONS HAUTES FRÉQUENCES. Journal de Physique IV Proceedings, 1992, 02 (C1), pp.C1-771-C1-774. 10.1051/jp4:19921168 . jpa-00251128

\section{HAL Id: jpa-00251128 https://hal.science/jpa-00251128}

Submitted on 1 Jan 1992

HAL is a multi-disciplinary open access archive for the deposit and dissemination of scientific research documents, whether they are published or not. The documents may come from teaching and research institutions in France or abroad, or from public or private research centers.
L'archive ouverte pluridisciplinaire HAL, est destinée au dépôt et à la diffusion de documents scientifiques de niveau recherche, publiés ou non, émanant des établissements d'enseignement et de recherche français ou étrangers, des laboratoires publics ou privés. 


\title{
CARACTERISATION DU SANG PAR ULTRASONS HAUTES FRÉUENCES
}

\author{
M. TOUBAL, E. RADZISZEWSKI, M. ASMANI, M. OURAK et B. NONGAILLARD \\ Laboratoire d'Opto-Acousto-Electronique (URA 832 CNRS), Université de Valenciennes, BP. 311, \\ F-59304 Valenciennes cedex, France
}

\begin{abstract}
Résumé: Nous nous sommes attachés dans ce travail, à déterminer expérimentalement l'atténuation des ultrasons dans le sang en fonction de ses constituants essentiels (plasma et globules rouges) dans une gamme de fréquences s'étendant de 50 à $400 \mathrm{MHz}$ à la température de $20^{\circ} \mathrm{C}$. L'originalité et l'intérêt d'une telle entreprise consistent en une prospection plus fine des caractéristiques acoustiques du sang étant donné que dans cette gamme de fréquences, les longueurs d'ondes deviennent comparables à la taille des diffuseurs (les globules rouges). Toutefois, le choix de l'atténuation en tant que moyen de caractérisation hautes fréquences n'est pas le plus judicieux, car il semblerait qu'il n'existe pas de corrélation remarquable entre la taille des diffuseurs et la longueur d'onde d'une part et l'atténuation mesurée avec un transducteur sensible à la phase d'autre part. Il sera nécessaire d'étudier l'évolution d'autres grandeurs telle que la répartition spatiale du diffusé en fonction de la fréquence. Des mesures de l'atténuation en fonction de l'hématocrite révèlent la même dépendance en fréquence des diverses courbes. L'atténuation montre une évolution croissante jusqu'à un hématocrite de $22.5 \%$, se stabilise entre $22.5 \%$ et $36 \%$ et évolue de nouveau jusqu'à $45 \%$. Il a également été mis en évidence que le plasma participe dans une proportion de $36 \%$ à l'atténuation dans le sang.
\end{abstract}

\begin{abstract}
Our interest here is in the experimental determination of ultrasonic attenuation in blood as a function of its main components (plasma and erythrocytes) in the frequency range between 50 and $400 \mathrm{MHz}$ at the room temperature of $20^{\circ} \mathrm{C}$. The originality of this work is that, with such high frequencies, the wavelengths become very close to the scatterers' ( the erythrocytes) size, leading to a microscopic characterization of the tissue. However, the choice of attenuation as a mean for high frequency characterization is not the most judicious one because it seems that no remarkable correlation exists between the scatterers' size and the ultrasonic wavelengths on one hand and the ultrasonic attenuation measured with a phase sensitive transducer on another hand. It will also prove necessary to study the variations of other physical parameters as the retrodiffused acoustic field spatial distribution versus frequency. The relation between the attenuation and the hematocrit is also examined up to a hematocrit of 45 percent. According to our findings, the attenuation increases along with the hematocrit until 22.5 percent, remains constant between 22.5 and 36 percent and then increases together with the hematocrit. We also measured a nearly 36 percent contribution of plasma to blood attenuation.
\end{abstract}

\section{I - INTRODUCTION}

La caractérisation des tissus biologiques demeure un problème sur lequel se penchent nombre de chercheurs à ce jour, étant donnée la complexité de l'interaction d'un faisceau ultrasonore avec une structure tissulaire même la plus simple. Depuis Cartensen [1a], l'un des précurseurs ayant montré la corrélation entre l'absorption ultrasonore et le patrimoine protéique du sang, la question des milieux biologiques présentant des particules en suspensions reste toujours posée. Le problème est abordé de deux points de vue, certains auteurs [1b,2] confrontant l'expérimentation avec des formalismes déjà existants, d'autres $[3,4,5]$ s'appuyant sur leur propre modélisation de l'interaction sons-tissus pour approcher les résultats expérimentaux. L'intérêt de la recherche s'est jusqu'à présent portée sur la caractérisation des tis- 
sus biologiques dans le domaine des fréquences médicales (1à $10 \mathrm{MHz})$, laissant celui des plus hautes fréquences à combler.

Pour notre part, il nous a semblé intéressant de commencer notre étude de la caractérisation ultrasonore hautes fréquences du sang par des mesures de l'atténuation en transmission.

\section{II- MATERIEL ET METHODE}

Les transducteurs utilisés, des pastilles piézoélectriques en niobate de lithium générant des ondes longitudinales (les seules pouvant se propager dans les milieux liquides), sont collées sur des lignes à retard en corindon de $10 \mathrm{~mm}$ de longueur. Un balayage sur toute la gamme de fréquence a nécessité l'utilisation de deux paires de sondes, de fréquences centrales respectives $100 \mathrm{MHz}$ et $300 \mathrm{MHz}$ et de bande passante relative égale à $100 \%$. La détermination de l'atténuation s'appuie sur une méthode de mesure en transmission et en impulsions. Un soin particulier a été apporté à la conception de la cuve expérimentale pour minimiser l'effet du parallélisme des faces. La mesure en relatif de l'atténuation est faite à partir d'atténuateurs calibrés en vertu de la décroissance exponentielle de l'amplitude d'une onde ultrasonore se propageant dans un milieu biologique.

A partir de $150 \mathrm{MHz}$, un étage amplificateur superhétérodyne a permis d'augmenter la sensibilité de la chaîne de mesure.

\section{III- EXPLOITATION DES RESULTATS}

Le sang a été rendu incoagulable par l'adjonction d' E.D.T.A., des cristaux de saponine en ont permis l'hémolyse.

\section{Calibration du système de mesure}

La calibration est faite dans l'eau dont l'atténuation est de $220 \mathrm{~dB} / \mathrm{mm} .(\mathrm{GHz})^{2}$ par une vérification de l'évolution quadratique de la courbe de l'atténuation en fonction de la fréquence.

2 Influence des protéines plasmatiques

Les mesures de l'absorption dans le plasma montre qu'à nos fréquences, une proportion de $36 \%$ de l'atténuation est due à cette phase liquide ( Fig. 1)

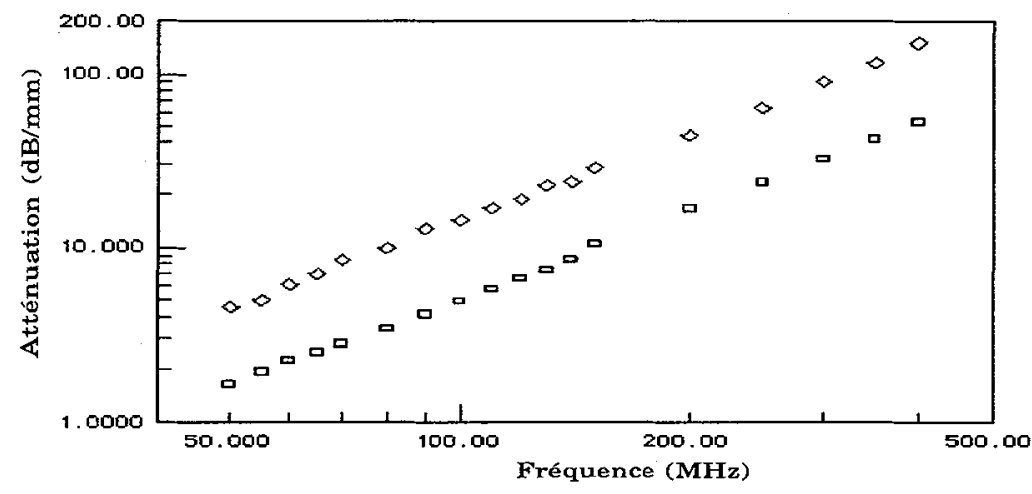

Fig. 1 : Influence du plasma sur l'atténuation $\diamond:$ sang total; $\square:$ plasma

\section{Influence des diffuseurs}

La diffusion est caractérisée par une variation d'amplitude, de phase, de vitesse et de direction de 
propagation de l'onde transmise par rapport à l'onde incidente. La section efficace de diffusion définie comme le rapport entre l'intensité de l'onde diffusée et de l'onde incidente est fonction de la relation entre obstacles et longueurs d'onde.

Aux fréquences auxquelles nous travaillons, et compte tenu que la vitesse dans le sang est de 1570 $\mathrm{m} / \mathrm{s}[6]$, les longueurs d'ondes sont comprises dans la fourchette [ $31.4-3.92 \mu \mathrm{m}]$.

Ceci signifie qu'à partir de $200 \mathrm{MHz}(\lambda=7.8 \mu \mathrm{m})$ la longueur d'onde devient comparable à la dimension du principal diffuseur qu'est le globule rouge.

\section{3-a quantification du diffusé dans la mesure de l'atténuation}

L'opération d'hémolyse a pour effet de supprimer les diffuseurs. L'atténuation que l'on mesure alors ne tient plus compte que de l'absorption dans le mélange plasma hémoglobine.

La comparaison avec l'atténuation mesurée dans le sang total montre que la proportion de diffusé est sensiblement la même dans toute la gamme de fréquences. La contribution du diffusé à l'atténuation qui serait donc indépendante du rapport de la longueur d'onde à la taille des diffuseurs est de l'ordre de $48 \%$ comme on peut le voir sur la figure 2.

\section{3-b mesure en fonction du nombre de diffuseurs}

Pour faire varier le nombre de diffuseurs, nous avons dilué le sang dans du plasma du même groupe sanguin. Cette opération équivalant à faire varier l'hématocrite (volume des globules rouges/ volume de sang total) a pour conséquence de modifier les caractéristiques physiques et chimiques du milieu (densité, viscosité, distribution des diffuseurs, distance inter-globulaire ...).

On peut remarquer qu'une modification de l'hématocrite ne change pas la dépendance en fréquence de l'atténuation (Fig. 3). Cependant, il est à noter l'influence de l'hématocrite puisque l'on observe une évolution croissante de la courbe d'atténuation aux fréquences comprises entre 150 et $400 \mathrm{MHz}$, ayant tendance à se stabiliser lorsque l'hématocrite se situe entre 22.5 et $36 \%$ (Fig. 4).
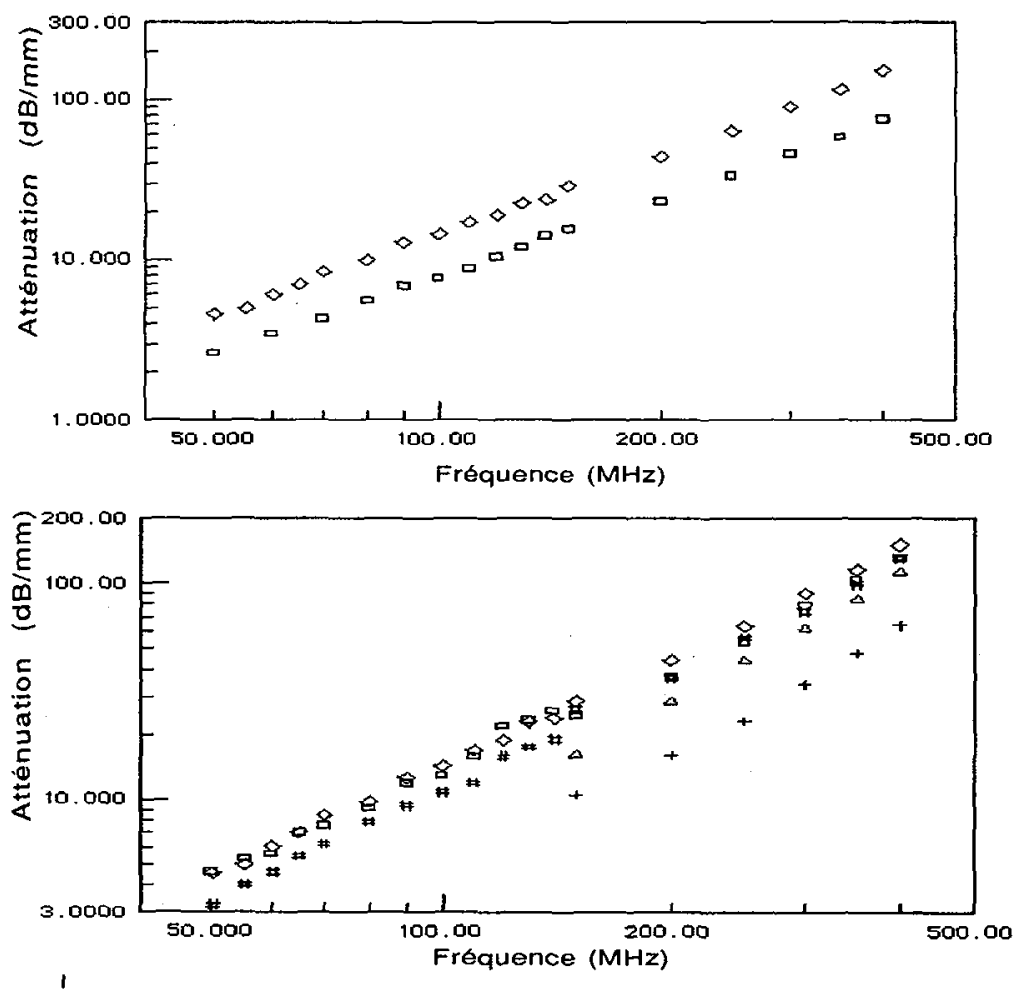

Fig. 2 : Influence de la diffusion sur l'atténuation

$\diamond$ : sang total; $\square$ : sang hémolysé

Fig. 3 : Influence des diffuseurs sur l'atténuation

$\diamond:$ hém. $45 \% ; \Delta$; hém.

$36 \%$; 口: hém. $22.5 \%$; \# : hém. $9 \%$; + : hém. $0.9 \%$ 


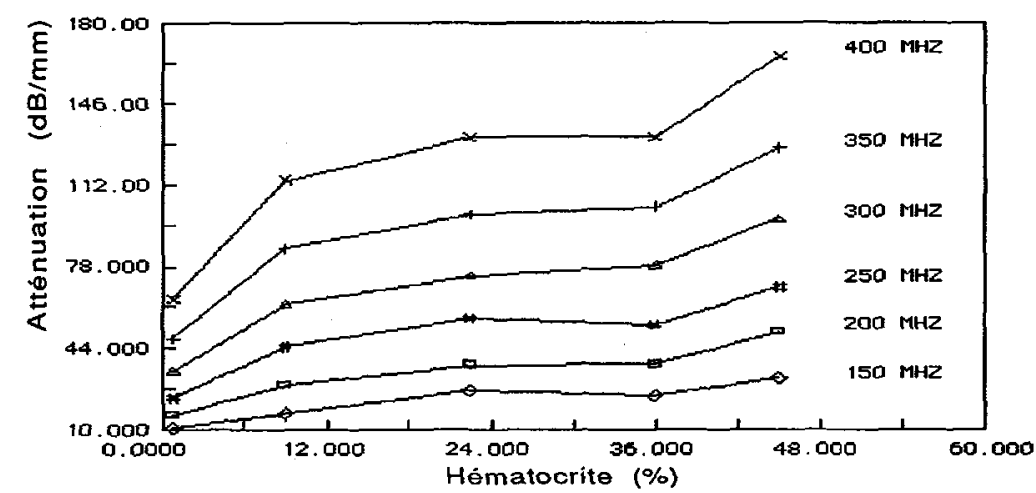

Fig. 4 : Influence de l'hématocrite sur l'atténuation

\section{CONCLUSION}

Ces résultats constituent une base pour les futurs travaux que nous comptons entreprendre concernant la caractérisation du sang à hautes fréquences.

Nous avons pu mettre au point un système de mesure fiable de l'atténuation en transmission, la précision de nos résultats étant inférieure à $10 \%$.

Nos travaux nous ont permis de montrer que le diffusé par les globules rouges participe pour près de la moitié à l'atténuation qui est également fonction de l'hématocrite.

C'est pourquoi dans une prochaine étape, nous allons nous intéresser à l'étude de la diffusion des ultrasons par les inhomogénéîtes afin de l'utiliser comme moyen de caractérisation.

\section{Remerciements}

Nous tenons à remercier le Centre de Biologie du Hainaut de bien vouloir nous fournir les échantillons de sang servant à nos études.

\section{REFERENCES}

[1] a- E. L. Cartensen, Kam Li, H. P. Schwan "Determination of the Acoustic Properties of Blood and its Components" J.A.S.A., vol. 25, 1953.

b- E. L. Cartensen, H. P. Schwan "Absorption of Sound Arising from the Precence of Intact Cells in Blood" J.A.S.A. , vol. 31, 1959.

[2] K. Shung, R. A. Sigelmann, J. M. Reid "Scattering of Ultrasound by Blood" IEEE Transactions on Biomedical Engineering, vol. BME-23, 1959.

[3] L. W. Anson, R.C. Chivers "Ultrasonic Propagation in Mammalian Cell Suspensions Based on a Shell Model" Phys. Med. Bio., 1989, vol.34, N 9, 1153-1167.

[4] U. Cobet "Modelling of Ultrasound Scattering By Blood" Journal of Nuclear Medicine \& Allied Sciences July. - Sept. 88.

[5] H. F. Routh, W. Cough, R. P. Williams "One Dimensional Computer Simulation of a Wave Incident on Randomly Distributed Inhomogeneities with Reference to the Scattering of Ultrasound by Blood" Med. \& Biol. Eng. \& Comput. , 1987, 25 667-671.

[6] J. P. Jones, S. Leeman "La Caractérisation des Tissus Biologiques par Ultrasons" Acta Electronica , 26, 1-2 , 1984, 3-31. 